\title{
Geochemical understanding of metallic species in the environmental matrices and strategic modifications of natural surfaces for aqueous sustainability
}

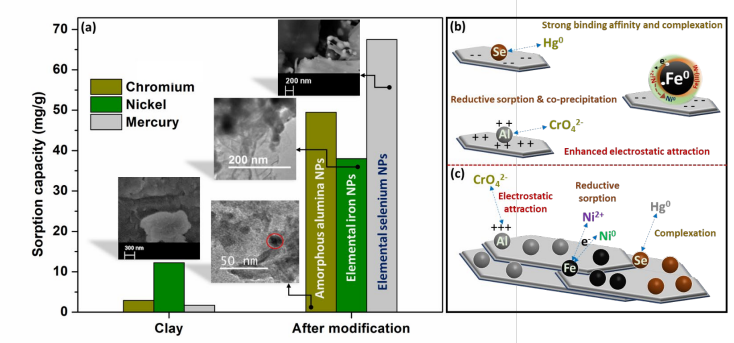

\author{
MR. NITIN KHANDELWAL, MS $^{1}$ AND GOPALA \\ KRISHNA DARBHA ${ }^{2}$ \\ ${ }^{1}$ Indian Institute of Science Education and Research (IISER)
} Kolkata

${ }^{2}$ IISER Kolkata

Presenting Author: nkk17ip016@iiserkol.ac.in

Aqueous reservoirs hold vast geochemical complexities in terms of water chemistry. Anthropogenic contamination of water bodies is a critical hurdle in achieving aqueous sustainability. Non-degradable toxic metals are one of the critical environmental concern. Based on the prevailing geochemical conditions, metals can speciate in various ionic forms such as$\mathrm{Ni}^{2+}, \mathrm{CrO}_{4}{ }^{2-}$ and $\mathrm{Hg}^{0}$. Most of the natural surfaces hold negative charge and therefore can uptake cationic metal species but in limited quantity. It shows the desire of strategic modifications of such natural surfaces to achieve efficient removal of toxicants.

We propose that detailed understanding of geochemical speciation of toxicants and prevailing geochemical parameters are crucial for developing an efficient adsorbent. To support the hypothesis, we have modified naturally abundant clay minerals through in-situ growth of (i) nanosized amorphous aluminum [1], (ii) elemental iron [2] and (iii) elemental selenium to handle anionic chromates, cationic metal species and elemental mercury respectively. Scientific basis lies on various fundamentals i.e. (i) clay provides a good surface for nanoparticle's growth, (ii) growth of alumina changes the surface charge of clays to positive causing enhanced electrostatic attraction of chromate ions, (iii) elemental iron can cause reduction and co-precipitation of metallic species and clays can minimize secondary contamination and (iv) strong $\mathrm{Se}-\mathrm{Hg}$ complexation can be achieved via growing $\mathrm{Se}^{0}$ nanoparticles on clay surface.

Results in fig. 1a clearly concludes that in comparison to natural clay surfaces, their strategic modification resulted in ultrafast and efficient sequestration of targeted toxic metal species through different sorption mechanisms as highlighted in fig. $1 \mathrm{~b}$ and $\mathrm{c}$. This suggests that a thorough geochemical understanding of adsorbate, adsorbent and prevailing matrices is critical in order to achieve aqueous sustainability.

Figure description

Fig. 1 (a) Toxicants sorption on clay before and after strategic modifications (morphological view in inset) and (b, c) schematics showing probable metallic species removal mechanisms

References

[1] N. Khandelwal, N. Singh, E. Tiwari, G.K. Darbha, Rsc Adv 9 (2019) 11160-11169.

[2] N. Khandelwal, E. Tiwari, N. Singh, G.K. Darbha, ACS ES\&T Water (2021). 